\title{
Overcoming Barriers in the Management of Lower Extremity Lymphedema Utilizing Advanced Pneumatic Therapy
}

\author{
Tina Hammond ${ }^{1}$ and Alison H. Golla*,2 \\ ${ }^{1}$ Washington Hospital Lymphedema Services, 2500 Mowry Avenue, Suite 140, Fremont, CA 94538, USA \\ ${ }^{2}$ Tactile Systems Technology, Inc., 1331 Tyler Street NE, Suite 200, Minneapolis, MN 55413, USA
}

\begin{abstract}
Lymphedema often requires intensive, costly treatment which initiates in the clinic and is eventually selfadministered by the patient at home. In-clinic Complete Decongestive Therapy aims to reduce limb volume, improve mobility, and reduce the risk of infection. Maintaining these clinical gains requires that the patient continues the treatments at home. Self-administering these treatments, however, can prove difficult, leading to loss of the therapeutic gains achieved during the in-clinic phase, especially when clinicians must moderate the recommendations to adapt to a patient's physical limitations and/or the demands of one's social and work obligations. Adding an advanced pneumatic therapy device in the home may assist with overcoming barriers associated with the complex requirements of selfadministered lymphedema care. By providing a consistent, passive treatment option, patients may be better able to maintain the clinical progress achieved during in-clinic therapy. The purpose of this retrospective case review was to assess whether adding such a device to the at-home treatment regimen made a difference in the patient's ability to manage lymphedema independently. This case study follows a patient with primary bilateral lower extremity lymphedema from initial in-clinic therapy through to her two year follow-up visit. Barriers to lymphedema management faced by this patient are identified, the clinical reasoning for the various treatment options tried is provided and the subsequent functional outcomes are presented. The case study suggests that the Flexitouch ${ }^{\circledR}$ system offers promise as an adjunct to in-home treatment for patients whose lymphedema has proven challenging to control.
\end{abstract}

\section{INTRODUCTION}

The lymphatic system plays a primary role in homeostasis by managing extracellular fluid volume, stabilizing interstitial protein concentration, and influencing tissue pressure [1]. The lymph system detoxifies and filters lymph fluid before returning what is needed by the body back to the general blood circulation. The lymphatic system is also vital in the defense against infection by its production of lymphocytes (white blood cells).

When the lymphatic system is compromised and cannot perform its primary role, the constituents of lymph fluid stagnate, resulting in lymphatic congestion which creates an environment conducive to infection. Build-up of lymphatic fluid begins in the interstitial space, triggering a chronic inflammatory response within the tissue as a futile surge of macrophages are directed to congested areas. Disorganized collagen fibers within the interstitium proliferate causing the progression of fibrotic changes in the tissue. Gradually the tissues indurate and become callous in a process called fibrosclerosis [1].

Without treatment to halt or reverse this progression, lymphedema worsens, leading to frequent bacterial and/or fungal infections, increasing size of the affected limb and adjacent trunk, functional impairment, and reduced quality of life [2]. Although there is currently no cure for

*Address correspondence to this author at the Tactile Systems Technology, Inc., 1331 Tyler Street NE, Suite 200, Minneapolis, MN 55413, USA; Tel: 612-355-5122; Fax: 612-355-5101; E-mail: agolla@tactilesystems.com lymphedema, treatment exists to lessen its severity and negative impact on a patient's life. Care and management of this chronic condition requires lifelong attention.

\section{LYMPHEDEMA STANDARD OF CARE}

The standard treatment for lymphedema is Complete Decongestive Therapy (CDT) performed in a clinic setting by a certified lymphedema therapist [3-5]. CDT uses a combination of the following components: manual lymph drainage (MLD) therapy, multi-layer short-stretch compression bandaging, therapeutic exercise, meticulous skin care, and lifelong use of compression garments. The goals of treatment are to reduce the limb weight/size, minimize the risk of infection, soften fibrotic tissue and improve limb function and mobility with subsequent maintenance of these clinical gains by the patient at home.

Lymph congestion can be drained by directing and moving the lymph fluid to functioning lymph vessels and nodes found in the adjacent truncal quadrant. Intact vessels joining both truncal territories, called anastomoses, are effectively utilized during decongestive treatment. MLD treatment uses a series of specialized hand movements, performed in a specific sequence to direct and move lymph fluid from an affected overloaded area (e.g. limb and trunk) along existing lymphatic pathways to functional adjacent lymph node groups. These functional lymph groups are stimulated first, preparing them to accept the additional fluid load from the affected extremity and to absorb and transport the fluid naturally with an eventual return to the circulatory system [1]. In addition to MLD, daily application of multi- 
layer short-stretch bandaging provides a therapeutic counterpressure gradient which facilitates lymph flow especially during muscle pump activity when the muscles in the affected area are activated (e.g. during therapeutic exercise) $[1,6]$. This technique decongests the affected area, softens fibrosity of the tissue, and remodels the limb contours. Specific decongestive exercises are performed while wearing the bandages to facilitate further lymph reabsorption. Meticulous skin care and adherence to precautions to prevent infection and edema exacerbation are also part of the treatment.

In-clinic CDT can last several weeks to months, depending upon the location, duration, and severity of the patient's lymphedema and can often be limited by third-party insurance coverage allowance. Effective long-term management of lymphedema requires that in-clinic treatment be followed by daily home maintenance therapy for the remainder of the patient's life. As a patient with lymphedema undergoes in-clinic treatment, therapists work to educate the patient on a home maintenance regimen and techniques for success. Maintaining the benefits of in-clinic therapy after discharge requires that the patient be able to effectively self-administer components of CDT on a daily basis.

\section{CHALLENGES TO HOME MAINTENANCE}

Although CDT is the standard of care with proven success for treating lymphedema in-clinic, implementing a successful home maintenance program is often a source of frustration for both the patient and therapist. When developing home maintenance regimens, therapists must consider the patient's physical and cognitive abilities, level of family support and assistance, financial implications, as well as the patient's proclivity to learn, adapt, and followthrough. The physical challenge of performing these techniques effectively at home requires range of motion, strength, and/or positioning that makes the treatments extremely difficult to self-administer. As a result, patients may experience deterioration in quality of life and loss of long-term treatment benefit [3]. Frequently, patients return to the therapist due to an exacerbation of symptoms following an inability to comply with the regimen of their current home program.

\section{NEW HOME TREATMENT OPTION}

In collaboration with the patient, therapists strive to create the best treatment regimen to successfully extend inclinic therapy gains and enhance home maintenance for each patient. Many have incorporated a device that treats lymphedema at home using advanced pneumatic compression technology. Using the Flexitouch ${ }^{\circledR}$ system (Tactile Systems Technology, Inc., Minneapolis, MN) at home can provide the benefits of MLD without requiring the patient to manually perform the techniques (Fig. 1). This multi-chamber, calibrated and programmable pneumatic compression device (PCD) was specifically designed to treat lymphedema. The system replicates the pressure magnitude, timing, and sequencing pattern that are uniquely compatible with the principles of MLD, and the physiology of lymph vessel absorption, transport and emptying [7]. In addition, this device, unlike older generation PCDs, treats and decongests the trunk, a key element in lymphedema treatment.

The Flexitouch system consists of an electronic controller unit connected by tubing harnesses to specially designed garments that wrap and contour around the affected limb and adjacent trunk. The unique sequencing of the 30-32 individual chambers, the work and release action of the gentle dynamic pressure, and its timing are all compatible with lymphatic anatomy and physiology and the principles of MLD. Treatment begins with individual chamber inflation and immediate deflation in peristaltic-like cycles of 1-3 second duration surrounding the trunk. The design of the garments and chambers link anastomoses between neighboring lymphatic drainage regions. The sequenced gradient pressure is applied to the truncal and more proximal areas first to prepare for the augmented flux of lymph towards intact lymph vessels; thereafter, lymph drainage of

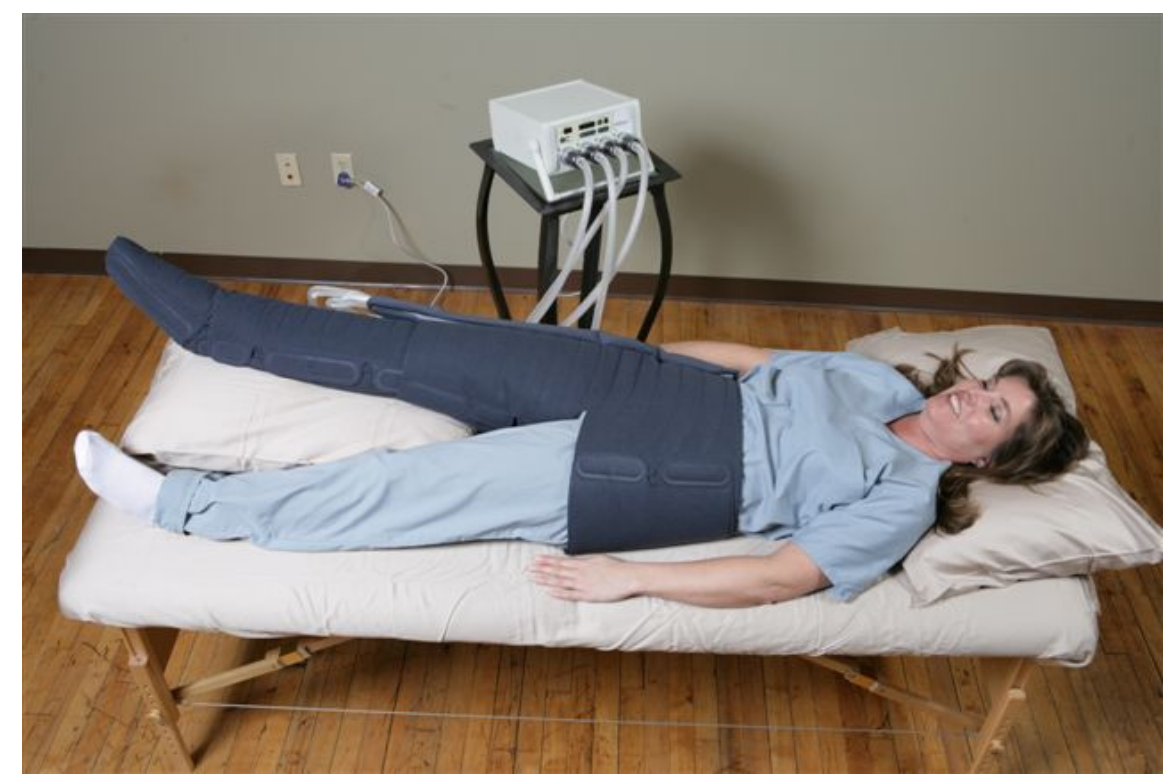

Fig. (1). The Flexitouch system for the lower extremity on a model. 
the affected extremity is accomplished by concerted cycles of pressure and release applied sequentially to the limb and trunk in a distal to proximal manner.

Emerging clinical evidence supports efficacy and acceptance of this advanced pneumatic therapy in assisting patients to achieve successful home management of their lymphedema. A pilot clinical trial found statistically significant limb volume reductions and weight loss in women with breast cancer-related lymphedema when using the Flexitouch system as compared to self MLD [3]. The study further reported the therapy was comfortable and wellaccepted by patients and the device easy to operate. A recent study evaluating 155 Flexitouch system users reported that $90 \%$ of the users were "satisfied" or "extremely satisfied" with the therapy; experienced clinically and statistically significant gains in physical and emotional health after using the system, and $95 \%$ were able to maintain or further reduce limb volume with Flexitouch use [2]. In addition to these findings, there was good patient adherence to therapy, further underscoring patient acceptance and implementation of this treatment into their home care regimen [2].

We believe that by providing a consistent, passive treatment option, that is specifically designed based on the established principles of manual lymphatic drainage, patients may be better able to maintain the clinical progress achieved during in-clinic therapy. The case study presented here assesses the practical and clinical implications of implementing this advanced therapy into the home treatment regimen for a patient with primary lower extremity lymphedema who had previously demonstrated difficulty with home treatment. This retrospective single case analysis compares the patient's outcomes to her own outcome over time; from the initial in-clinic evaluation through to the two year follow up visit. The outcome measures reported include: 1) changes in limb volume and 2) observed and patient reported functional gains. The patient provided written consent to allow publication of her data. All limb volumes were measured and calculated in the clinic utilizing an infrared optoelectronic system (Pero- System, PeroPlus 2000; Juzo, Inc, Cuyahoga Falls, OH). The validity and reliability of the Perometer for limb volume measurement has been established [8-11].

\section{PATIENT DESCRIPTION}

The patient was a 53 year-old female with a history of bilateral swelling in her lower extremities. Initially, the swelling would appear and then resolve but over time the swelling became persistent. She sought medical treatment, but was undiagnosed for several years. The swelling was uncontrolled and getting worse every year. Eventually, she was referred to a cardiovascular physician at Stanford University who diagnosed her with severe primary (congenital) lymphedema affecting the bilateral lower extremities and trunk. In-clinic lymphedema therapy was prescribed. At initiation of acute lymphedema treatment she exhibited numerous functional deficits. Table 1 summarizes the functional deficits, the CDT protocol and the initial recommended home program.

\section{CLINICAL PROBLEM SOLVING}

The patient was treated in clinic by a certified lymphedema therapist 3 times per week for 90 minute sessions for 4 weeks. Instruction in self-bandaging was deemed not viable because the patient was unable to reach her feet and her spouse also had physical limitations that precluded use of this modality at home. In lieu of bandaging, the therapist recommended bilateral knee-high CircAid compression systems be worn daily over $20-30 \mathrm{mmHg}$ kneehigh compression garments. The combination of these worn together provided the level of compression necessary to prevent re-accumulation as she was not able to independently don compression stockings of a higher level

\section{Table 1. Functional Deficits and CDT Program}

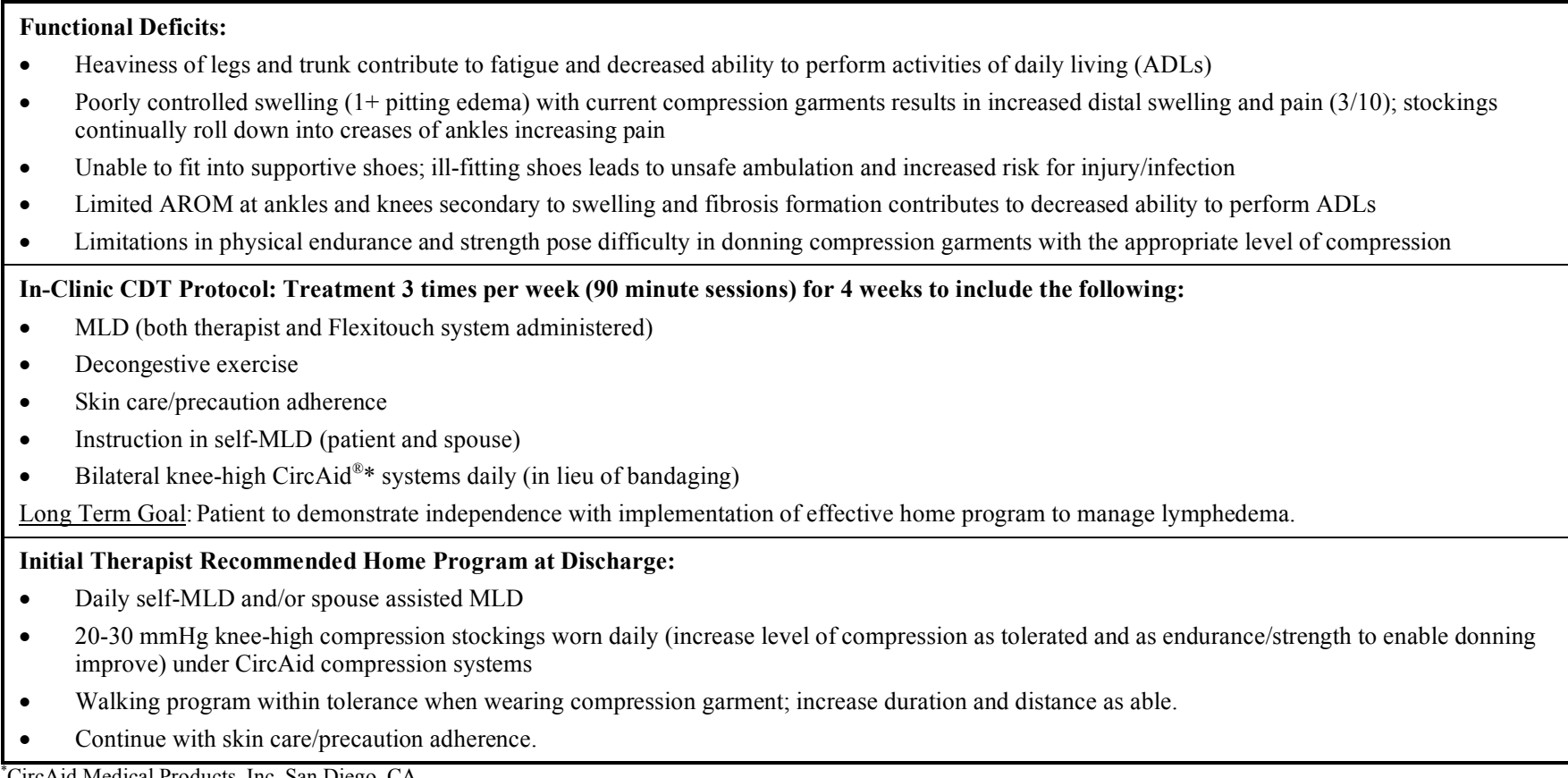

CircAid Medical Products, Inc, San Diego, CA. 
compression. After 4 weeks, the patient was discharged with a home maintenance program. At the conclusion of the four weeks (total of 12 treatments) of in-clinic therapy, the patient achieved the following therapy goals:

- Limb volume reduction of $4 \%$ bilaterally

- Pain reduction (0/10) bilaterally in legs and feet

- Independence with self-MLD supplemented by spouse administered MLD

- Independence in donning and tolerance for daily wear of compression garments (bilateral knee-high CircAid systems and 20-30 $\mathrm{mmHg}$ knee-high compression stockings)

- Verbalization of lymphedema precautions and demonstration of skin care to prevent infection and exacerbation of symptoms

\section{ONE MONTH FOLLOW-UP}

At one month post discharge, she was reassessed by her therapist. At that time, the patient reported daily compliance with self and spouse assisted MLD, daily compression stocking wear, and a short distance walking program. She had, however, discontinued use of the CircAid systems because she could not keep them from slipping down throughout the day which required frequent readjusting. Her limb volume had increased by $2.5 \%$ in her left leg and $2.1 \%$ in her right leg. The patient reported that the positive results of self and/or spouse administered MLD at home were very short-term and the procedures for carrying out these routines were impractical and time-consuming. The MLD treatment alone took over an hour to complete on each leg. In addition, the self-MLD required her to be isolated from her family and friends for the duration of the treatment due to the personal nature of the physical requirements and positioning and the level of mental concentration it required. Moreover, the swelling would reaccumulate within a few hours. The pain $(3 / 10)$ in her feet returned as they became more swollen. Despite compliance to the current recommended protocols of home therapy, the patient and therapist were disappointed with the outcomes of her self-management regimen. The patient questioned her ability to comply with the demands of the regimen in light of the limited results she was obtaining, especially when considering the need for lifelong management.

The goal of successful transition to an effective home program was not achieved. Self and spouse assisted MLD at home in combination with compression garment wear was not sufficient to control her bilateral lower extremity lymphedema. Implementation of the Flexitouch system for one hour treatment sessions per leg each day, 3 times per week was recommended as an adjunct to the current home regimen. The Flexitouch system was selected to augment the patient's home program over other pneumatic devices based on the expectation that its conformance with the physiology of the lymphatic system [7] would better facilitate decongestion and lymph mobilization and avoid risks associated with older generation PCDs [1, 12-15]. After inclinic training, the patient demonstrated independence with Flexitouch system garment donning and device operation. She initiated use of the Flexitouch system as part of her home program.

\section{TWO MONTH FOLLOW-UP}

- $\quad$ Patient reports compliance with Flexitouch treatment 3 times per week for 1 hour (per limb) and continued daily 20-30 mmHg compression garment wear.

- $\quad$ Patient reported ability to tolerate being up on feet for increased durations of time and began using Masai Balance Technology (MBT) (Masai Marketing \&Trading AG, Switzerland) shoes during her routine walking program.

- Re-assessment in-clinic showed essentially stable limb volume ( $\mathrm{L}$ leg increased by $0.8 \%$ and $\mathrm{R}$ leg by $0.02 \%$ when compared to limb volume at time of discharge from in-clinic CDT).

Patient demonstrated good compliance with home program and limb volumes were stable with miniscule changes. Two months prior to this date, the patient initiated taking a prescription drug to assist with blood sugar control for diabetes. Swelling was a side effect of the medication, which may have inhibited further progress toward controlling edema. The patient reported reduced pain in her feet, and increased duration with walking program while wearing MBT shoes, which based on limited reports on patients with orthopedic impairments, are believed to enhance biomechanical effects during walking, and may decrease pain and improve calf musculature [16-18]. Patient reported she is able to incorporate Flexitouch system treatments while doing other meaningful activities, such as visiting with family and friends. In other words, it did not isolate her or necessitate physical positions and/or exposure that would be inappropriate in the presence of others. In addition, she found it to be more efficient as it was able to treat a much larger surface area in the same period of time as self-administered therapy. Most importantly, she found the treatment to be more effective and the results longer lasting in comparison to that of self-MLD, as such, she complied more readily.

\section{ROUTINE ONE YEAR FOLLOW-UP}

- Patient demonstrated bilateral limb volume reduction (L leg reduced by $3.8 \%$ and $\mathrm{R}$ leg by $6.3 \%$ ).

- $\quad$ Patient reports compliance with Flexitouch treatment 3 times per week for 1 hour (per limb) and continued daily 20-30 mmHg compression garment wear.

- $\quad$ Patient had gradually increased walking program to a total of 1.5 miles $(2.42 \mathrm{~km})$ per week.

- Improvement in physical stamina and pain $(0 / 10)$ to allow completion of all daily activities.

- Patient discontinued need for use of prescription medication for diabetes 6 months prior and diabetes was well controlled.

Patient continued to demonstrate good bilateral limb volume control with additional reduction. Long-term goal of effective home program implementation was achieved.

\section{ROUTINE TWO YEAR FOLLOW-UP}

- Patient continued to demonstrate limb volume reduction bilaterally (L leg volume reduced by $7.9 \%$ and $\mathrm{R}$ leg by $10.8 \%$ ). 
- Patient continued to report consistency with Flexitouch use and has eliminated the need for self and spouse assisted MLD altogether.

- $\quad$ As a result of the girth reduction, patient could now tolerate and easily don $30-40 \mathrm{mmHg}$ compression garments to prevent re-accumulation of fluid.

- $\quad$ Patient continued to increase duration and distance of walking program to a total of 5 miles per week.

- No exacerbation of lymphedema symptoms or need for in-clinic therapy within the past two years.

The patient demonstrated substantial bilateral limb volume reduction since discharge from acute in-clinic CDT two years prior. Fig. (2) graphs the limb volume reduction over time. Before and after photos are included in Figs. (3, 4). She continued to successfully implement her home program and had no exacerbations of her lymphedema. The patient continues to be satisfied with her outcomes and home management program.

\section{DISCUSSION}

This patient incorporated use of the Flexitouch system as an adjunct to an existing home care regimen after experiencing disappointing outcomes, including an increase in bilateral limb volume, in as little as one month after discharge from an intensive in-clinic CDT program. In less than 4 weeks of daily therapy with the Flexitouch, limb volumes had reduced to near discharge levels. Using the Flexitouch regularly, her limb volumes continued to reduce and by the two-year follow up visit, limb volumes had reduced by almost $8 \%$ on the more affected left leg and almost $11 \%$ on the right leg. The patient attributed the reduced swelling and pain to enabling her to build endurance and strength by incrementally increasing the duration and distance of her walking program. As a result of the concomitant functional gains, she became increasingly able to complete all ADLS independently without hindrance from fatigue, pain or impaired ROM. Meeting her own personal goals of reduced reliance on professional administered therapy, she resumed an active lifestyle. Most importantly for the patient, she gained a sense of control over her condition and now feels equipped to manage this condition long-term.

Although, the patient attributes the gains she has made directly to use of the Flexitouch, it is not known how much utilizing the MBT shoes contributed to her overall comfort during walking and subsequent gains in stamina and strength. However, the patient believes it was the use of the Flexitouch that enabled her to reduce her limb girth sufficiently to allow for a proper and comfortable fit of the MBT shoes which in turn allowed her to comply with the walking program. Consistent therapeutic exercise (i.e. increased muscle pump activity) in combination with the Flexitouch likely contributed to her limb volume reduction.

We believe that compliance is key to success of any home management program for patients with lymphedema. As such, it is the responsibility of the therapist in collaboration with the patient to develop a suitable home program that can be sustained by the patient long-term. Given the complex demands of home therapy, the clinical

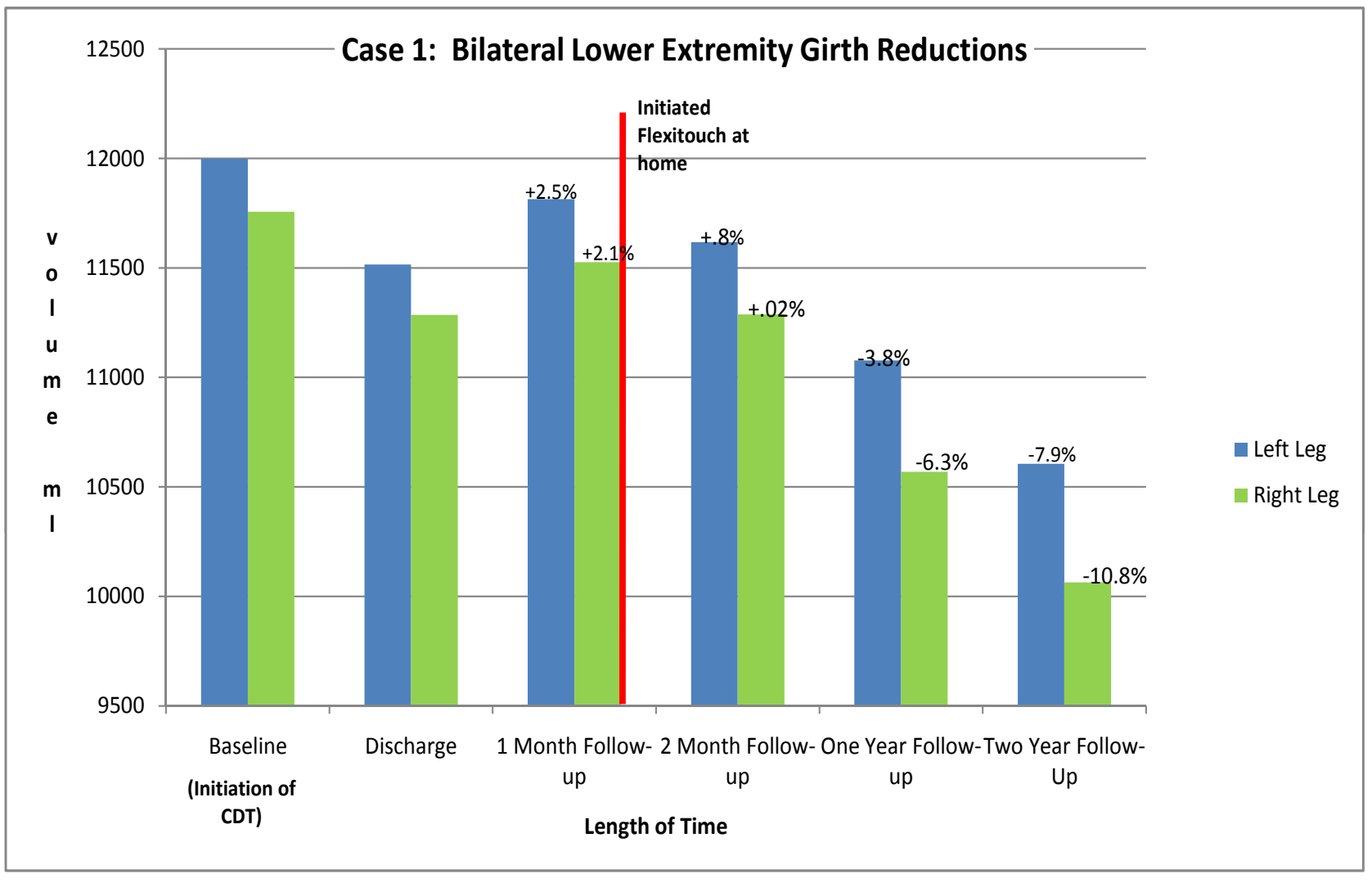

Fig. (2). Bilateral limb volume reductions over time as compared to limb volume at discharge from in-clinic CDT. 


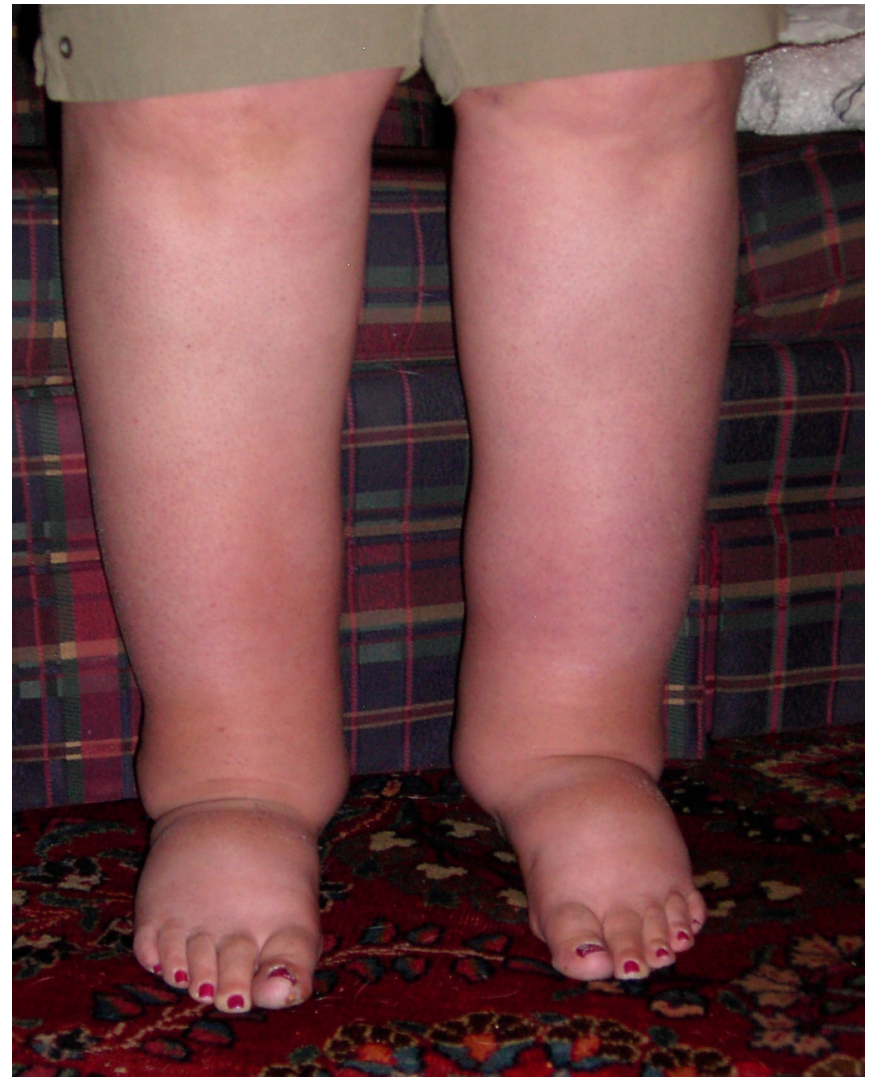

Fig. (3). Patient at beginning of CDT treatment.

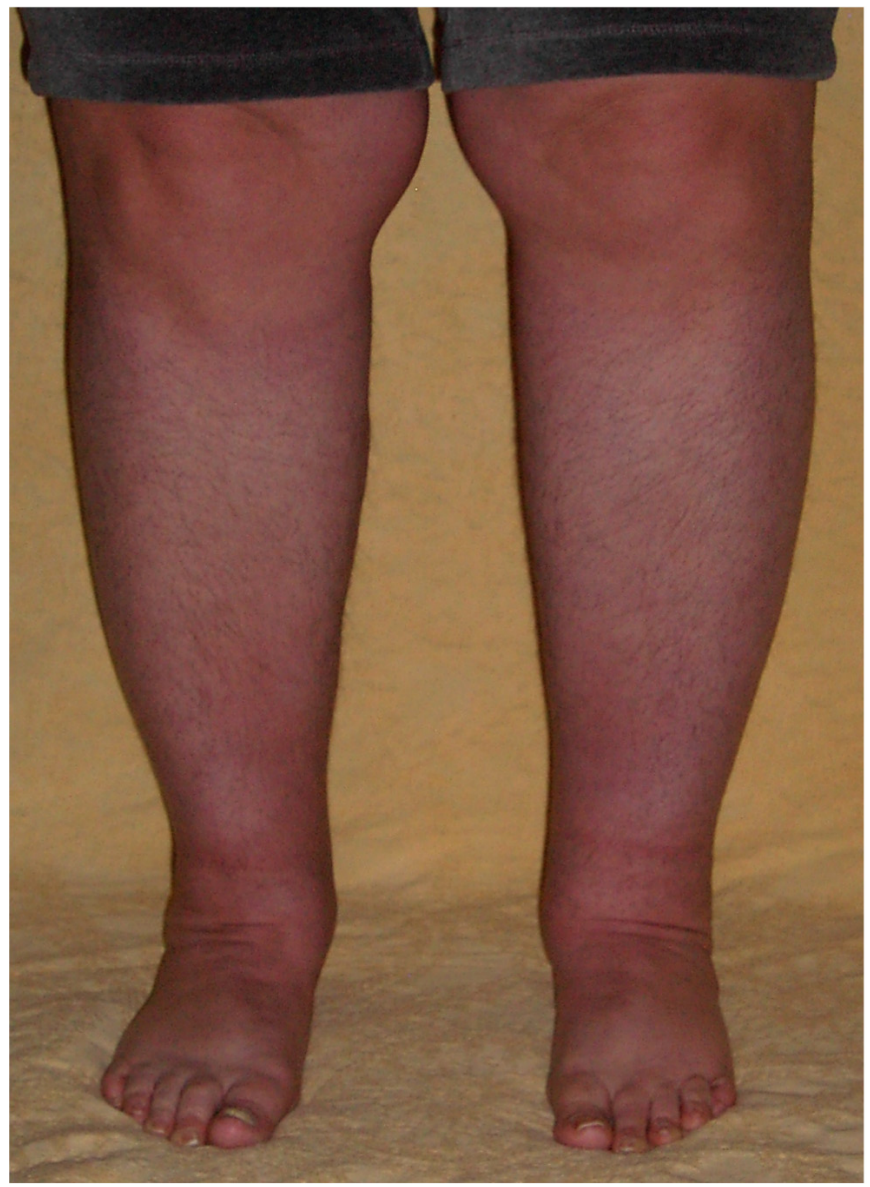

Fig. (4). Patient at 2 year follow-up. problem solving in determining a suitable home program is often challenging. Patients are likely to adhere to therapy protocols in direct proportion to their perception of benefit weighed against treatment-associated effort, discomfort and unpleasantness. When at home treatment fails to lead to satisfactory outcomes, a major contributing factor is the considerable time and effort required to achieve often disappointing, and/or temporary results. This patient experienced clinical benefit and a high level of satisfaction which likely contributed to her willingness to integrate its use into her routine treatment regimen. Furthermore, it instilled in her a sense of control while taking the burden off to fully master and perform the complex manual techniques of self-MLD, likely improving the effectiveness of at-home treatment in general. As Wilburn and associates [3] suggested, since this device, designed to simulate the manual techniques of MLD, provides consistent, automated therapy, it should curtail the effects of improper or inadequate technique. The outcome experienced by the patient presented here is consistent with the findings of Ridner et al. [5] in that satisfaction with the device, and perceived clinical benefit supported sustained compliance with the therapy. Strengths of this case report are the length of time this patient was followed and the corresponding changes in limb volume, functional status, and level of independence obtained with her home program. A limitation of the study is that it is a single patient review.

\section{CONCLUSION}

Lymphedema is a chronic, often debilitating condition which requires interminable lifelong management. Insufficient treatment leads to exacerbations of swelling and pain, recurrent infections, progressive tissue hardening or fibrosis, and continued loss of mobility and function. For some, this becomes an onerous cycle necessitating multiple rounds of in-clinic therapy and/or increasing medical complications. While therapists can rely on the established and sound principles of CDT treatment in the clinic, patients cannot continue costly and inconvenient in-clinic treatment on a long-term basis.

In the transition to at-home management however, patients encounter various barriers to effective treatment. As this case example has shown, at-home treatments can be arduous and are not always feasible for patients to adhere to. The challenges faced by this patient are not uncommon. The discovery, assessment and implementation of new ways to successfully extend in-clinic therapy gains and enhance home maintenance are vital to optimal patient care and clinicians should be resourceful and creative in trying to bridge that gap.

By administering therapy based on the anatomy and physiology of the lymphatic system and the well-established methods and principles of MLD, the Flexitouch system may offer a safe and effective treatment to maintain and often further reduce limb volumes long term. This advanced pneumatic device is especially suited for patients who are in pursuit of alternatives to frequent in-clinic visits or those who have encountered challenges with more traditional home maintenance programs. The Flexitouch system may be a beneficial addition to at-home treatment plans to sustain health, facilitate compliance, and improve quality of life in 
light of this chronic, progressive disease that is undoubtedly complicated by the normal aging process. Could this device serve a person throughout their lifetime? A prospective, randomized, crossover trial with patients who have demonstrated difficulty with long-term home maintenance is warranted to further assess the clinical outcomes, level of compliance, quality of life and cost-effectiveness of utilizing this advanced PCD long-term. Also, beneficial would be further studies that are qualitative in nature that ruminate the multifarious factors of patient engagement and compliance in home treatment protocols and how those factors may incite outcomes. For the patient described here, inclusion of this passive treatment, allowed her to meet her functional goals, gain a sense of control of her lymphedema and return to an active and satisfying lifestyle.

\section{ABBREVIATIONS}

$$
\begin{aligned}
& \text { CDT }=\text { Complete Decongestive Therapy } \\
& \text { MLD }=\text { Manual Lymphatic Drainage } \\
& \text { PCD }=\text { Pneumatic Compression Device } \\
& \text { ADLs }=\text { Activities of Daily Living } \\
& \text { AROM }=\text { Active Range of Motion } \\
& \text { mmHg }=\text { Millimeters of mercury pressure } \\
& \mathrm{R} \\
& \mathrm{L}
\end{aligned}
$$

\section{ACKNOWLEDGEMENT}

We would like to acknowledge and thank the patient for allowing us to share her story.

\section{CONFLICT OF INTEREST}

Alison H. Golla is employed by Tactile Systems, Inc., which provided assistance with preparation of this manuscript.

\section{REFERENCES}

[1] Foeldi M, Foeldi E. Textbook of lymphology for physicians and lymphedema therapists. Munich, Germany: Urban and Fischer 2006.

[2] Ridner SH, McMahon E, Dietrich MS, Hoy S. Home-based lymphedema treatment in patients with cancer-related lymphedema or noncancer-related lymphedema. Oncol Nurs Forum 2008; 35: 671-80.

[3] Wilburn O, Wilburn P, Rockson SG. A pilot, prospective evaluation of a novel alternative for maintenance therapy of breast cancer-associated lymphedema. BMC Cancer 2006; 6: 84.

[4] Position statement of the National Lymphedema Network; NLN treatment position paper [Online]. 2006. Available from: http:// www.lymphnet.org/pdfDocs/nlntreatment.pdf

[5] International Society of Lymphology Executive Committee. The diagnosis and treatment of peripheral lymphedema. Lymphology 2003; 36: 84-91.

[6] Chikley B. Silent waves: theory and practice of lymph drainage therapy. Scottsdale, Arizona: International Health \& Healing Inc. Publishing 2001.

[7] Mayrovitz HN. Interface pressures produced by two different types of lymphedema therapy devices. Phys Ther 2007; 87: 1379-88.

[8] Stanton A, Northfield J, Holroyd B, Mortimer P, Levick J. Validation of an optoelectronic limb volumeter (Perometer). Lymphology 1997; 30: 77-97.

[9] Mayrovitz H, Sims N, Macdonald J. Assessment of limb volume by manual and automated methods in patients with limb edema or lymphedema. Adv Skin Wound Care 2000; 13: 272-6.

[10] Tierney A, Aslam M, Rennie K, Grace P. Infrared optoelectronic volumetry, the ideal way to measure limb volume. Eur J Vasc Endovasc Surg 1997; 14: 415-6.

[11] Man IO, Markland KL, Morrissey MC. The validity and reliability of the Perometer in evaluating human knee volume. Clin Physiol Funct Imaging 2004; 24(6): 352-8.

[12] Cheville AL, McGarvey CL, Petrek JA, Russo SA, Taylor ME, Thiadens SR. Lymphedema management. Semin Radiat Oncol 2003; 13: 290-301.

[13] Eliska O, Eliska M. Are peripheral lymphatics damaged by high pressure massage? Lymphology 1995; 28: 21-30.

[14] Boris M, Weindorf S, Lasinski BB. The risk of genital edema after external pump compression for lower limb lymphedema. Lymphology 1998; 31: 15-20.

[15] Miranda F, Perez M del CJ, Castiglioni MLV, et al. Effect of sequential intermittent pneumatic compression on both leg lymphedema volume and on lymph transport as semi-quantitatively evaluated by lymphoscintigraphy. Lymphology 2001; 34: 135-41.

[16] Kraus I, Bendig A, Mayer J, Axmann D, Müller O, Horstmann T. Effect of a 10 week training program with a novel training device in patients with osteoarthritis of the hip $(\mathrm{AOH})$ or after total hip replacement on health related quality of life. Dtsch Z Sportmed 2006; 57: 195-200.

[17] Nigg B, Hintzen S, Ferber R. Effect of an unstable shoe construction on lower extremity gait characteristics. Clin Biomech 2006; 21: 82-8

[18] Gollhofer A. Functional testing of the MBT shoe compared to sensorimotor training [Online]. 2007 Aug 1. Available from: http://www.swissmasaius.com/Science

This is an open access article licensed under the terms of the Creative Commons Attribution Non-Commercial License (http://creativecommons.org/licenses/by-nc/ 3.0/) which permits unrestricted, non-commercial use, distribution and reproduction in any medium, provided the work is properly cited. 